\title{
2-Dimensional Reversible Hexagonal Cellular Automata with Periodic Boundary
}

\author{
S. UGUZ ${ }^{a}$, I. SiAP ${ }^{b}$ AND H. AKIN ${ }^{c}$ \\ ${ }^{a}$ Department of Mathematics, Arts and Science Faculty, Harran University, Sanliurfa, 63120, Turkey \\ ${ }^{b}$ Department of Mathematics, Yildiz Technical University, 34210, Istanbul, Turkey \\ ${ }^{c}$ Department of Mathematics, Education Faculty, Zirve University, 27260, Gaziantep, Turkey
}

\begin{abstract}
In this paper, we study 2-dimensional finite cellular automata defined by hexagonal local rule with periodic boundary over the field $Z_{3}$. We construct the rule matrix corresponding to the hexagonal cellular automata. For some given coefficients and the number of columns of hexagonal information matrix, we prove that the hexagonal cellular automata are reversible.
\end{abstract}

DOI: 10.12693 /APhysPolA.123.480

PACS: 02.10.Yn, 07.05.Kf, 02.10.Ox

\section{Introduction}

The hexagonal finite cellular automata (shortly HFCA) are 2-dimensional (2D) cellular automata whose cells are of the form of hexagonal. Morita et al. [1] introduced this type of cellular automaton $(\mathrm{CA})$ and they called it hexagonal partitioned CA (HPCA). A remarkable application of the family of these CAs is presented by Trunfio [2] where a model is presented to simulate the evolution of forest fires and Hernández Encinas et al. [3] where they introduce a new mathematical model for predicting the spread of a fire front in homogeneous and inhomogeneous environments. Also, in [4], debris flows are simulated and modeled by two-dimensional hexagonal cellular automata. These families of cellular automata are also applied to design discrete models of chemical reaction-diffusion systems [5].

Also, 2D CAs have found applications in traffic modeling. For instance multi-value (including ternary) CA models for traffic flow are proposed in [6]. Recently, cellular automata have found applications in cryptography $[7,8]$, especially 2D CA have been proposed for multi-secret sharing scheme for colored images [9].

Due to the applications and modeling on hexagonal cellular automata, the algebraic structure of cellular automata has been of much interest to the researchers [10-13]. Algebraic representation of 2D CA helps in determining the characterization of CA. An important characterization is the determination of the reversibility of CA [13]. In [14], we have characterized a 2D finite CA by using matrix algebra built on $Z_{3}$. Also, we have analyzed some results about the rule numbers $2460 \mathrm{~N}$ and 2460P. In [15], we have obtained necessary and sufficient conditions for the existence of Garden of Eden configurations for $2 \mathrm{D}$ ternary CAs.

In this paper, we deal with $\mathrm{CA}$ defined by hexagonal rules under periodic boundary condition $(\mathrm{PBC})$ and the ternary field $Z_{3}$. We obtain the rule matrix of the hexagonal finite periodic cellular automaton (HFPCA). We compute the rank of rule matrices related to HFPCA via an algorithm. Hence, we determine the reversibility of this type 2D CA which is one of the difficult problems in higher dimension as explained in the previous paragraph. Further, by using the matrix algebra it is shown that the HFPCA are reversible, if the number of columns is even and the HFPCA are not reversible, if the number of the columns is odd.

A periodic boundary $\mathrm{CA}$ is the one where the extreme cells in the boundaries are adjacent to each other periodically [16]. A null boundary CA is the one where the extreme cells in the boundaries are connected to the zero states. The surrounding cells are all in zero state. For convenience of analysis, the state of each cell is an element of a finite or infinite state set. Moreover, the state of the cell $(i, j)$ at time $t$ is denoted by $x_{(i, j)}^{(t)}$. The state of the cell $(i, j)$ at time $t+1$ is denoted by $x_{(i, j)}^{(t+1)}=y_{(i, j)}^{(t)}$. Let us consider the

$$
C^{(t)}=\left(\begin{array}{ccc}
x_{11}^{(t)} & \ldots & x_{1 n}^{(t)} \\
\vdots & \ddots & \vdots \\
x_{m 1}^{(t)} & \ldots & x_{m n}^{(t)}
\end{array}\right) .
$$

The matrix $C^{(t)}$ is called the configuration of the 2D finite $\mathrm{CA}$ at time $t$. We associate planar hexagonal presentations with column vectors by transforming them from $C^{(t)}$ to $\left([X]_{m n \times 1}\right)^{\mathrm{T}}=$ $\left(x_{11}^{(t)}, x_{12}^{(t)}, \ldots, x_{1 n}^{(t)}, \ldots, x_{m 1}^{(t)}, \ldots, x_{m n}^{(t)}\right)^{\mathrm{T}}$.

Hence, we can consider the transition matrix $T_{R}$ such that $\left(T_{R}\right)_{m n \times m n}[X]_{m n \times 1}=[Y]_{m n \times 1}$, where $\left([Y]_{m n \times 1}\right)^{\mathrm{T}}=\left(y_{11}^{(t)}, y_{12}^{(t)}, \ldots, y_{1 n}^{(t)}, \ldots, y_{m 1}^{(t)}, \ldots, y_{m n}^{(t)}\right)^{\mathrm{T}}$. If $j$ is an even positive integer, then we have

$$
\begin{aligned}
& y_{(i, j)}^{(t)}=a x_{(i-1, j)}^{(t)}+b x_{(i, j+1)}^{(t)}+c x_{(i+1, j+1)}^{(t)}+d x_{(i+1, j)}^{(t)} \\
& \quad+e x_{(i+1, j-1)}^{(t)}+f x_{(i, j-1)}^{(t)} \bmod 3 .
\end{aligned}
$$

If $j$ is an odd positive integer, then we have

$$
\begin{aligned}
& y_{(i, j)}^{(t)}=a x_{(i-1, j)}^{(t)}+b x_{(i-1, j+1)}^{(t)}+c x_{(i, j+1)}^{(t)} \\
& \quad+d x_{(i+1, j)}^{(t)}+e x_{(i, j-1)}^{(t)}+f x_{(i-1, j-1)}^{(t)} \bmod 3,
\end{aligned}
$$


where $a, b, c, d, e, f \in Z_{3}^{*}=Z_{3} \backslash\{0\}=\{1,2\}$ and $x_{(i, j)}^{(t)} \in Z_{3}$.

\section{The rule matrix}

In this section, we obtain the rule matrix corresponding to the 2D finite HCAs defined by hexagonal local rules.

Theorem 1: Let $a, b, c, d, e, f \in Z_{3}^{*}, m \geq 3$ and $n$ be an even positive integer. Then, under periodic boundary condition the rule matrix $\left(T_{R}^{0}\right)_{m n \times m n}$ from $Z_{3}^{m n}$ to $Z_{3}^{m n}$ which takes the $t$-th finite hexagonal configuration $C^{(t)}$ of order $m \times n$ to the $(t+1)$-th state $C^{(t+1)}$ is given by

$$
\left(T_{R}^{0}\right)_{m n \times m n}=\left(\begin{array}{cccccccc}
A^{0} & B^{0} & O & O & \ldots & \ldots & O & C^{0} \\
C^{0} & A^{0} & B^{0} & O & \ldots & \ldots & O & O \\
O & C^{0} & A^{0} & B^{0} & O & \ldots & O & O \\
\vdots & \vdots & \vdots & \vdots & \vdots & \vdots & \vdots & \vdots \\
O & O & \ldots & O & C^{0} & A^{0} & B^{0} & O \\
O & O & \ldots & \ldots & O & C^{0} & A^{0} & B^{0} \\
B^{0} & O & \ldots & \ldots & O & O & C^{0} & A^{0}
\end{array}\right) \text {, }
$$

where each submatrix is of order $n \times n$ and $O$ is zero matrix of order $n \times n$,

$$
\begin{aligned}
A^{0}= & \left(\begin{array}{ccccccc}
0 & c & 0 & 0 & \ldots & 0 & e \\
f & 0 & b & 0 & \ldots & 0 & 0 \\
0 & e & 0 & c & \ldots & 0 & 0 \\
0 & 0 & f & 0 & \ldots & 0 & 0 \\
\vdots & \vdots & \vdots & \vdots & \ddots & \vdots & \vdots \\
0 & 0 & \ldots & 0 & e & 0 & c \\
b & 0 & \ldots & 0 & 0 & f & 0
\end{array}\right), \\
B^{0} & =\left(\begin{array}{ccccccc}
d & 0 & 0 & 0 & \ldots & 0 & 0 \\
e & d & c & 0 & \ldots & 0 & 0 \\
0 & 0 & d & 0 & \ldots & 0 & 0 \\
0 & 0 & e & d & \ldots & 0 & 0 \\
\vdots & \vdots & \vdots & \vdots & \ddots & \vdots & \vdots \\
0 & 0 & \ldots & 0 & 0 & d & 0 \\
c & 0 & \ldots & 0 & 0 & e & d
\end{array}\right), \\
C^{0} & =\left(\begin{array}{ccccccc}
a & b & 0 & 0 & \ldots & 0 & f \\
0 & a & 0 & 0 & \ldots & 0 & 0 \\
0 & f & a & b & \ldots & 0 & 0 \\
0 & 0 & 0 & a & \ldots & 0 & 0 \\
\vdots & \vdots & \vdots & \vdots & \ddots & \vdots & \vdots \\
0 & 0 & \ldots & 0 & f & a & b \\
0 & 0 & \ldots & 0 & 0 & 0 & a
\end{array}\right),
\end{aligned}
$$

Theorem 2: Let $a, b, c, d, e, f \in Z_{3}^{*}, m \geq 3$ and $n$ be an odd positive integer. Then, under periodic boundary condition the rule matrix $\left(T_{R}^{1}\right)_{m n \times m n}$ from $Z_{3}^{m n}$ to $Z_{3}^{m n}$ which takes the $t$-th finite hexagonal configuration $C^{(t)}$ of order $m \times n$ to the $(t+1)$-th state $C^{(t+1)}$ is given by

$$
\left(T_{R}^{1}\right)_{m n \times m n}=\left(\begin{array}{cccccccc}
A^{1} & B^{1} & O & O & \ldots & \ldots & O & C^{1} \\
C^{1} & A^{1} & B^{1} & O & \ldots & \ldots & O & O \\
O & C^{1} & A^{1} & B^{1} & O & \ldots & O & O \\
\vdots & \vdots & \vdots & \vdots & \vdots & \vdots & \vdots & \vdots \\
O & O & \ldots & O & C^{1} & A^{1} & B^{1} & O \\
O & O & \ldots & \ldots & O & C^{1} & A^{1} & B^{1} \\
B^{1} & O & \ldots & \ldots & O & O & C^{1} & A^{1}
\end{array}\right),
$$

where each submatrix is of order $n \times n$ and $O$ is zero matrix of order $n \times n$,

$$
A^{1}=\left(\begin{array}{ccccccc}
0 & c & 0 & 0 & \ldots & 0 & e \\
f & 0 & b & 0 & \ldots & 0 & 0 \\
0 & e & 0 & c & \ldots & 0 & 0 \\
0 & 0 & f & 0 & \ldots & 0 & 0 \\
\vdots & \vdots & \vdots & \vdots & \ddots & \vdots & \vdots \\
0 & 0 & \ldots & 0 & f & 0 & b \\
c & 0 & \ldots & 0 & 0 & e & 0
\end{array}\right)
$$

$$
B^{1}=\left(\begin{array}{ccccccc}
d & 0 & 0 & 0 & \ldots & 0 & 0 \\
e & d & c & 0 & \ldots & 0 & 0 \\
0 & 0 & d & 0 & \ldots & 0 & 0 \\
0 & 0 & e & d & \ldots & 0 & 0 \\
\vdots & \vdots & \vdots & \vdots & \ddots & \vdots & \vdots \\
0 & 0 & \ldots & 0 & e & d & c \\
0 & 0 & \ldots & 0 & 0 & 0 & d
\end{array}\right)
$$

$$
C^{1}=\left(\begin{array}{ccccccc}
a & b & 0 & 0 & \ldots & 0 & f \\
0 & a & 0 & 0 & \ldots & 0 & 0 \\
0 & f & a & b & \ldots & 0 & 0 \\
0 & 0 & 0 & a & \ldots & 0 & 0 \\
\vdots & \vdots & \vdots & \vdots & \ddots & \vdots & \vdots \\
0 & 0 & \ldots & 0 & 0 & a & 0 \\
b & 0 & \ldots & 0 & 0 & f & a
\end{array}\right) .
$$

The theorem can be proved similar to the theorem in $[13]$.

\section{The reversibility cases of $2 \mathrm{D}$ HFPCAs}

As mentioned in introduction, the problem of determining whether a $2 \mathrm{D} \mathrm{CA}$ is reversible is a very difficult problem in general. Further, there is no algorithm in general [17]. In this section, we make use of the structure obtained in the previous sections and determine the reversibility problem for all orders $m \times n$. In order to present the problem and its solution we need to split the problem in two parts depending on the integer $m$ whether 
it is even or odd. We will conclude that if the rule matrix associated to the $\mathrm{HFPCA}_{m \times n}$ is invertible, then the $\mathrm{HFPCA}_{m \times n}$ is reversible, otherwise, the $\mathrm{HFPCA}_{m \times n}$ is irreversible. To determine the invertibility of the rule matrix associated to the $\mathrm{HFPCA}_{m \times n}$, we first study the submatrix $A^{0}$ for even case.

\subsection{The rank of $T_{R}^{0}$}

Lemma 1: For all even $n=2 k$ with odd $k>1$, $\operatorname{det}\left(A_{2 k}^{0}\right)=-\left(c^{k}+\mathrm{e}^{k}\right)\left(b^{k}+f^{k}\right)$. Since $b, c, e, f$ are all non-zero, then $A_{2 k}^{0}$ (with odd $k$ ) is invertible.

Lemma 2: For all even $n=2 k$ with even $k$, $\operatorname{det}\left(A_{2 k}^{0}\right)=-\left(c^{k}-\mathrm{e}^{k}\right)\left(b^{k}-f^{k}\right)$. Hence

(i) If $c \neq e$ and $b \neq f$, then $A_{2 k}^{0}$ (with even $k$ ) is invertible,

(ii) If $c=e$ or $b=f$, then $A_{2 k}^{0}$ (with even $k$ ) is non-invertible.

Hence, when $n$ is even, the determinant of matrix $A_{n}^{0}$ is not non-zero for all $k$, so another method for determining the reversibility of the $\mathrm{CA}$ is needed. This method that presents an algorithm for determining the rank of the rule matrix is given in the sequel. To determine the reversibility of the $\mathrm{CA}$, we study the determinant of the submatrices $B_{n}^{0}$ and $C_{n}^{0}$.

Lemma 3: For all even $n$, if the matrix $B_{n}^{0}$ is given as Eq. (1), then $\operatorname{det}\left(B_{n}^{0}\right)=d^{n}$. Since, $d \neq 0, B_{n}^{0}$ is invertible.

Lemma 4: For all even $n$, if the matrix $C_{n}^{0}$ is given as Eq. (1) then $\operatorname{det}\left(C_{n}^{0}\right)=a^{n}$. Since $a \neq 0, C_{n}^{0}$ is invertible.

Let $T_{i}$ denote the $i$-th row and $T_{i}[j]$ denote the $j$-th entry of the $i$-th row of matrix $T$, respectively. By definition, we have

$$
\begin{aligned}
T_{1}= & {\left[A^{0}, B^{0}, 0,0,0,0, \ldots, 0, C^{0}\right] \in M_{n \times n}\left(Z_{3}\right)^{m}, } \\
T_{2}= & {\left[C^{0}, A^{0}, B^{0}, 0,0,0,0, \ldots, 0\right] \in M_{n \times n}\left(Z_{3}\right)^{m}, } \\
& \vdots \quad \vdots \\
T_{m}= & {\left[B^{0}, 0,0,0,0,0, \ldots, C^{0}, A^{0}\right] \in M_{n \times n}\left(Z_{3}\right)^{m} . }
\end{aligned}
$$

Define the $\sigma$ map as follows: $\sigma: M_{n \times n}\left(Z_{3}\right)^{m} \rightarrow$ $M_{n \times n}\left(Z_{3}\right)^{m} . \quad \sigma\left(\left[A_{1}, A_{2}, A_{3}, \ldots, A_{m-1}, A_{m}\right]\right)=$ $\left[0_{n}, A_{1}, A_{2}, A_{3}, \ldots, A_{m-1}\right]$ where $0_{n}$ represents the zero square matrix of order $n$. Further, if $A=\left[A_{1}, A_{2}, A_{3}, \ldots, A_{m-1}, A_{m}\right]$, then $A[i]=A_{i}$ represents the $i$-th entry of $A$. Further, $B\left[A_{1}, A_{2}, \ldots, A_{m}\right]=\left[B A_{1}, B A_{2}, \ldots, B A_{m}\right]$.

Theorem 3 (Even): Let the matrix $\left(T_{R}^{0}\right)_{m n \times m n}$ be given as Eq. (1) and

$$
\begin{aligned}
& T_{1}^{(1)}=T_{1}, T_{1}^{(k+1)}=-T_{1}^{(k)}[k]\left(C^{0}\right)^{-1} \sigma^{(k-1)}\left(T_{2}\right) \\
& \quad+T_{1}^{(k)} \quad \text { for } \quad 1 \leq k \leq m-2, \\
& T_{m}^{(1)}=T_{m}, T_{m}^{(k+1)}=-T_{m}^{(k)}[k]\left(C^{0}\right)^{-1} \sigma^{(k-1)}\left(T_{2}\right) \\
& \quad+T_{m}^{(k)} \text { for } 1 \leq k \leq m-2 .
\end{aligned}
$$

Define the following $2 \times 2$ block matrix consisting of blocks of square matrices of order $n$.

$$
Q^{0}=\left(\begin{array}{ll}
T_{1}^{(m-1)}[m-1] & T_{1}^{(m-1)}[m] \\
T_{m}^{(m-1)}[m-1] & T_{m}^{(m-1)}[m]
\end{array}\right) .
$$

Then,

$$
\operatorname{rank}\left(T_{R}^{0}\right)_{m n \times m n}=(m-2) n+\operatorname{rank}\left(Q^{0}\right) .
$$

A straightforward corollary which gives a lower bound for the rank of a cellular automaton is presented below.

Corollary 1: Let $\left(T_{R}^{0}\right)_{m n \times m n}$ be a cellular automaton defined above. Then, $(m-2) n \leq \operatorname{rank}\left(\left(T_{R}^{0}\right)_{m n \times m n}\right) \leq$ mn.

\subsection{The rank of $T_{R}^{1}$}

Lemma 5: If $n \geq 3$ is odd (choose $n=2 k+1$ ), then $\operatorname{det}\left(A_{n=2 k+1}^{1}\right)=b^{k} c^{k+1}+\mathrm{e}^{k+1} f^{k}$. Since $b, c, e, f$ are all non-zero, $A_{n}^{1}$ is invertible.

Lemma 6: For all odd $n$, if the matrix is given as Eq. (2) then $\operatorname{det}\left(B_{n}^{1}\right)=d^{n}$. Since $d \neq 0, B_{n}^{1}$ is invertible.

Lemma 7: If $n \geq 3$ is odd (choose $n=2 k+1$ ), then $\operatorname{det}\left(C_{n=2 k+1}^{1}\right)=a^{2 \bar{k}-1}\left(a^{2}-b f\right)$. If $a^{2} \neq b f$ then $C_{n}^{1}$ is invertible.

By using the result from the fundamental theorem of linear algebra, the rank of a matrix is equal to the rank of its transpose, i.e. $\operatorname{rank}\left(T_{R}^{1}\right)=\operatorname{rank}\left(\left(T_{R}^{1}\right)^{\mathrm{T}}\right)$. Then, the matrix $T_{R}^{1}$ can be viewed as follows for easy computation of its rank. A matrix and its transpose have the same determinant. This implies that $\left(A^{1}\right)^{\mathrm{T}}$ and $\left(B^{1}\right)^{\mathrm{T}}$ are also full rank matrices. Let us now choose $T_{i}$ for the rank computation $T_{R}^{1}$ as follows.

$$
\begin{gathered}
T_{1}=\left[\left(A^{1}\right)^{\mathrm{T}},\left(C^{1}\right)^{\mathrm{T}}, 0,0, \ldots, 0,\left(B^{1}\right)^{\mathrm{T}}\right] \in M_{n \times n}\left(Z_{3}\right)^{m}, \\
T_{2}=\left[\left(B^{1}\right)^{\mathrm{T}},\left(A^{1}\right)^{\mathrm{T}},\left(C^{1}\right)^{\mathrm{T}}, 0, \ldots, 0,0\right] \in M_{n \times n}\left(Z_{3}\right)^{m}, \\
\vdots \quad \vdots \\
T_{m}=\left[\left(C^{1}\right)^{\mathrm{T}}, 0, \ldots, 0,0,\left(B^{1}\right)^{\mathrm{T}},\left(A^{1}\right)^{\mathrm{T}}\right] \in M_{n \times n}\left(Z_{3}\right)^{m} .
\end{gathered}
$$

Hence we obtain the following result.

Theorem 4 (Odd). Let the matrix $\left(T_{R}^{1}\right)_{m n \times m n}$ be given as in Eq. (2). Let

$$
\begin{aligned}
& T_{1}^{(1)}=T_{1}, T_{1}^{(k+1)}=-T_{1}^{(k)}[k]\left(\left(B^{1}\right)^{\mathrm{T}}\right)^{-1} \sigma^{(k-1)}\left(T_{2}\right) \\
& \quad+T_{1}^{(k)} \text { for } 1 \leq k \leq m-2, \\
& T_{m}^{(1)}=T_{m}, T_{m}^{(k+1)}=-T_{m}^{(k)}[k]\left(\left(B^{1}\right)^{\mathrm{T}}\right)^{-1} \sigma^{(k-1)}\left(T_{2}\right) \\
& \quad+T_{m}^{(k)} \text { for } 1 \leq k \leq m-2 .
\end{aligned}
$$

Define the following $2 \times 2$ block matrix consisting of blocks of square matrices of order $n$

$$
Q^{1}=\left(\begin{array}{ll}
T_{1}^{(m-1)}[m-1] & T_{1}^{(m-1)}[m] \\
T_{m}^{(m-1)}[m-1] & T_{m}^{(m-1)}[m]
\end{array}\right) .
$$

Then, we have $\operatorname{rank}\left(T_{R}^{1}\right)_{m n \times m n}=(m-2) n+\operatorname{rank}\left(Q^{1}\right)$.

\section{Conclusion}

In this paper, we have studied 2-dimensional finite cellular automata defined by hexagonal local rule with periodic boundary over the field $Z_{3}$. We have constructed the rule matrix corresponding to the hexagonal cellular automata. For some given coefficients and the number of columns of hexagonal information matrix, 
we have proved that the hexagonal cellular automata are reversible. We are planning to give some applications relating to these cellular automata over the prime field $F_{p}$, $p$ is a prime number. Our future work will continue in this direction.

\section{Acknowledgments}

This work is supported by TUBITAK, project number: $110 \mathrm{~T} 713$.

\section{References}

[1] K. Morita, M. Margenstern, K. Imai, RAIRO-Theor. Inf. Appl. 33, 535 (1999)

[2] G.A. Trunfio, Lecture Notes Comput. Sci. 3305, 385 (2004)

[3] L. Hernández Encinas, S. Hoya White, A. Martín del Rey, G. Rodríguez Sánchez, Appl. Math. Model. 31, 1213 (2007)

[4] D. D'Ambrosio, S. Di Gregorio, G. Iovine, Nat. Hazards Earth Syst. Sci. 3, 545 (2003)

[5] A. Adamatzky, A. Wuensche, B. De Lacy Costello, Chaos Solutions Fractals 27, 287 (2006)

[6] K. Nishinar, D. Takahashi, J. Phys. A Math. Gen. 33, 7709 (2000)
[7] S.R. Blackburn, S. Murphy, K.G. Peterson, IEEE Trans. Comput. 46, 637 (1997)

[8] A. Fuster-Sabater, P. Caballero-Gil, Appl. Math. Lett. 22, 1518 (2009)

[9] G. Alvarez, L. Hernández Encinas, A. Martín del Rey, Inf. Sci. 178, 4382 (2008)

[10] G. Alvarez Maranon, L. Hernandez Encinas, A. Hernandez Encinas, A. Martin del Rey, G. Rodriguez Sanchez, Lecture Notes in Comput. Sci. 2773, 1207 (2003)

[11] C. Fraile Rubio, L. Hernandez Encinas, S. Hoya White, A. Martin del Rey, G. Rodriguez Sanchez, Neural Parallel Sci. Comput. 12, 175 (2004).

[12] N. Romero, A. Rovella, F. Vilamajo, Appl. Math. Lett. 19, 576 (2006)

[13] I. Siap, H. Akın, S. Uguz, Comput. Math. Appl. 62, 4161 (2011)

[14] I. Siap, H. Akın, F. Sah, J. Frank. Inst. 348, 1258 (2011)

[15] I. Siap, H. Akın, F. Sah, Inf. Sci. 180, 3562 (2010)

[16] Z. Cinkir, H. Akın, I. Siap, J. Stat. Phys. 143, 807 (2011)

[17] J. Kari, Physica D 45, 386 (1990) 